\title{
Ability of the rhTSH stimulation test to predict relapse in patients with differentiated thyroid carcinoma, after long-term follow-up
}

\author{
MAFALDA MARCELINO ${ }^{1,2}$, ANA FILIPA LOPES ${ }^{1}$, DEOLINDA MADUREIRA $^{3}$, \\ TERESA C. FERREIRA ${ }^{4}$, EDWARD LIMBERT $^{1}$ and VALERIANO LEITE ${ }^{1}$
}

${ }^{1}$ Department of Endocrinology, Portugese Institute of Oncology, Lisbon 1600-608; ${ }^{2}$ Department of Endocrinology, Armed Forces Hospital, Lisbon 1649-020; ${ }^{3}$ Laboratory of Endocrinology and ${ }^{4}$ Department of Nuclear-Medicine, Portugese Institute of Oncology, Lisbon 1600-608, Portugal

Received January 14, 2014; Accepted August 12, 2014

DOI: $10.3892 / \mathrm{ol} .2015 .2854$

\begin{abstract}
The analysis of serum thyroglobulin ( $\mathrm{Tg}$ ) following thyroid-stimulating hormone (TSH) stimulation (sTg) has been recommended in the follow-up of differentiated thyroid carcinoma (DTC) patients, however, its routine use remains controversial. The aim of the current study was to evaluate the accuracy of sTg testing following recombinant human (rh) TSH stimulation in DTC patients, with a follow-up of 12.4 years. Retrospective studies were conducted of 125 DTC patients, who underwent rhTSH stimulation testing between 1999 and 2002. The exclusion criteria were: Patients with anti-Tg antibodies, Tg levels $>1 \mathrm{ng} / \mathrm{ml}$ under TSH suppression and the absence of radioactive iodine (RAI) ablation therapy following surgery. In total, 49 patients were included in the study and all had been previously treated with total or near total thyroidectomy (with or without central neck dissection) and RAI, postoperatively. The Tg functional sensitivity was $1.0 \mathrm{ng} / \mathrm{ml}$. The follow-up for patients was performed annually. During the median follow-up of 12.4 years after the rhTSH stimulation test, nine patients exhibited recurrence (18.4\%). Of the nine patients, six exhibited sTg levels $>2 \mathrm{ng} / \mathrm{ml}$ (positive result) and three exhibited levels $<2 \mathrm{ng} / \mathrm{ml}$ (negative result). Relapse occurred at a mean of 5.9 years following the rhTSH stimulation test. The positive predictive value and negative predictive value (NPV) of positive sTg were 50 and $91.9 \%$, respectively, with a sensitivity of $66.6 \%$ and a specificity of $85.0 \%$. The rhTSH-stimulated Tg levels have a high NPV, allowing the identification of the patients who are free of
\end{abstract}

Correspondence to: Dr Mafalda Marcelino, Department of Endocrinology, Portuguese Institute of Oncology, Rua Professor Lima Basto, Lisbon 1099-023, Portugal

E-mail: marcelino.mafalda@gmail.com

Key words: recurrence, follow-up, differentiated thyroid carcinoma, rhTSH test, accuracy the tumour. These results are consistent with the previously published data; however, to the best of our knowledge, this is the study with the longest follow-up duration after rhTSH stimulation.

\section{Introduction}

Thyroid carcinoma comprises 1-2\% of all malignancies in the USA (1). Epidemiological studies have shown an increasing prevalence of differentiated thyroid carcinoma (DTC); however the mortality rate has remained stable, most likely due to the increased diagnostic scrutiny (2). Over half of the mortalities in the USA result from papillary carcinoma, the majority of which are low-risk tumours (3). Among DTC patients, $\sim 80 \%$ exhibit no evidence of disease following the initial treatment (4). However, the recurrence rate is $10-30 \%$ (depending on the initial therapy), and the cancer may reappear after several years (up to one-third of cases recur after the first decade) $(5,6)$, indicating a requirement for prolonged follow-up.

According to the American Thyroid Association recommendations (7), the disease-free status comprises all of the following: i) No clinical evidence of tumour; ii) no imaging evidence of tumour [i.e. no uptake outside the thyroid bed on the initial post-treatment whole-body scan (WBS) or, if uptake outside the thyroid bed is present, no imaging evidence of tumour on a recent diagnostic scan and neck ultrasound (US) is observed]; and iii) undetectable serum thyroglobulin ( $\mathrm{Tg}$ ) levels during thyroid-stimulating hormone (TSH) suppression and stimulation in the absence of interfering antibodies.

The same guidelines also suggest that initial follow-up for low-risk patients should be based predominantly on TSH-suppressive serum Tg (supTg) and neck US, followed by $\mathrm{TSH}$-stimulated serum $\mathrm{Tg}$ (sTg) measurements if the supTg is undetectable (7). However, ultrasensitive methods for serum thyroglobulin determination may be used to avoid TSH stimulation 9-12 months following surgery in low-risk patients who have an undetectable serum thyroglobulin on levothyroxine (LT4) treatment (8). This was supported by Brassard et al (4), who published a prospective study of 715 DTC patients and 
concluded that in the majority of patients $(84 \%)$ who exhibited low supTg levels $(<0.27 \mathrm{ng} / \mathrm{ml})$, TSH stimulation did not increase the negative predictive value of Tg determination on the risk of recurrence (99\% in the two conditions). In this context, recent publications suggested that negative sTg in the initial approach has such a high negative predictive value, that future sTg are questionable $(9,10)$, and even the initial stimulation test may be replaced by a more sensitive $\mathrm{Tg}$ assay (8). Another predictive factor of persistent or recurrent DTC, with high negative predictive value (NPV) and that has been studied in recent years, is the Tg level at the time of recombinant human (rh) TSH-aided ablation, which may be used as a prognostic marker (11).

As the majority of the studies published thus far have a short-term follow-up after the rhTSH stimulation test ( $\leq 7$ years), and recurrence may occur in up to one-third of cases after the first decade, the present study aimed to evaluate the accuracy of rhTSH-stimulated Tg levels in patients with undetectable supTg values (on LT4 therapy), to predict the remission after a follow-up of 12.4 years.

\section{Patients and methods}

Patient selection. Between 1999 and 2002, the determination of Tg levels following rhTSH stimulation testing was routinely performed in low-risk DTC patients at the Department of Endocrinology at the Portugese Institute of Oncology (Lisbon, Portugal). This study was approved by the ethics committee of the Portugese Institute of Oncology and written informed consent was obtained from all patients.

In total, 125 DTC patients were evaluated who underwent a total or near total thyroidectomy, which was completed by central neck dissection in 38\% of cases, resulting in the apparent complete resection of the neoplastic tissue. Overall, $88 \%$ of these patients underwent radioactive iodine (RAI) ablation therapy postoperatively. For all but one patient, no focus of uptake outside the thyroid bed was detected on the WBS, performed 2 days following ${ }^{131} \mathrm{I}$ therapy. Subsequently, all patients were treated with suppressive LT4 doses. Tumour stages were classified according to the TNM scoring system (12). Exclusion criteria for this study were: i) presence of anti-Tg antibodies; ii) $\mathrm{TSH}$-suppressive $\mathrm{Tg}$ levels $>1 \mathrm{ng} / \mathrm{ml}$; and iii) absence of RAI ablation therapy following surgery. Following the application of these criteria, 49 patients remained in the study (Fig. 1), who were divided in two groups according to: i) Positive sTg levels $>2 \mathrm{ng} / \mathrm{ml}$ and ii) negative sTg levels $<2 \mathrm{ng} / \mathrm{ml}$.

Procedures. Patients were given $0.9 \mathrm{mg}$ rhTSH intramuscularly on two consecutive days, followed by $4 \mathrm{mCi}{ }^{131} \mathrm{I}$ after $24 \mathrm{~h}$. Additionally, the serum Tg measurement and diagnostic WBS (DxWBS) were conducted on day 5 ( $72 \mathrm{~h}$ following the final rhTSH injection).

DxWBS was classified as follows: i) negative when no uptake was observed or in the case of neck iodine uptake consistent with a normal thyroid remnant; or ii) suspicious in the case of uptake outside the thyroid bed, suggesting underlying disease. The $\mathrm{Tg}$ measurements were obtained using manual immunoradiometric Tg assays, (SELco TG kits; Medipan GmbH, Selchow, Germany) with a functional
Table I. Characteristics of the 49 patients at presentation.

\begin{tabular}{|c|c|c|}
\hline \multirow[b]{2}{*}{ Characteristics } & \multicolumn{2}{|c|}{ Patients $(n=49)$} \\
\hline & $\mathrm{n}$ & $\%$ \\
\hline \multicolumn{3}{|l|}{ Gender } \\
\hline Female & 41 & 83.7 \\
\hline Male & 8 & 16.3 \\
\hline Mean age, years & \multicolumn{2}{|c|}{60.6} \\
\hline \multicolumn{3}{|l|}{ TNM classification (12) } \\
\hline $\mathrm{T} 1$ & 4 & 8.2 \\
\hline $\mathrm{T} 2$ & 10 & 20.4 \\
\hline $\mathrm{T} 3$ & 9 & 18.4 \\
\hline $\mathrm{T} 4$ & 7 & 14.3 \\
\hline $\mathrm{Tx}$ & 19 & 38.8 \\
\hline \multicolumn{3}{|l|}{ Lymph nodes } \\
\hline No & 21 & 42.9 \\
\hline N1 & 22 & 44.9 \\
\hline $\mathrm{Nx}$ & 6 & 12.2 \\
\hline \multicolumn{3}{|l|}{ Distant metastasis } \\
\hline M0 & 48 & 98.0 \\
\hline M1 & 1 & 2.0 \\
\hline \multicolumn{3}{|l|}{ Stage } \\
\hline I & 28 & 57.1 \\
\hline II & 6 & 12.2 \\
\hline III & 5 & 10.2 \\
\hline IV & 1 & 2.0 \\
\hline Unknown & 9 & 18.4 \\
\hline \multicolumn{3}{|l|}{ Histology } \\
\hline Papillary & 44 & 89.8 \\
\hline Follicular & 4 & 8.2 \\
\hline Papillary + follicular & 1 & 2.0 \\
\hline \multicolumn{3}{|l|}{ Surgery type } \\
\hline Total thyroidectomy & 24 & 49.0 \\
\hline Near total thyroidectomy & 6 & 12.2 \\
\hline $\begin{array}{l}\text { Total thyroidectomy with } \\
\text { central neck dissection }\end{array}$ & 19 & 38.8 \\
\hline
\end{tabular}

sensitivity of $1 \mathrm{ng} / \mathrm{ml}$. Based on the functional sensitivity of the assay, a concentration of $1 \mathrm{ng} / \mathrm{ml}$ was selected as the cut-off value discriminating undetectable from detectable $\mathrm{Tg}$ levels.

Follow-up. Follow-up assessments were conducted annually and consisted of a clinical examination, serum supTg determination under LT4 treatment and occasionally, neck US. Patients were categorised as 'no evidence of disease' (NED) if the neck US was negative and if all supTg levels were $<1.0 \mathrm{ng} / \mathrm{ml}$. Persistent tumours were identified by fine needle aspiration cytology (when the neck US was positive) or by ${ }^{1311}$ WBS showing ${ }^{131}$ I uptake outside the thyroid bed. Chest computed tomography was performed when the neck US was positive or when the supTg levels were $>1.0 \mathrm{ng} / \mathrm{ml}$ with negative US. 

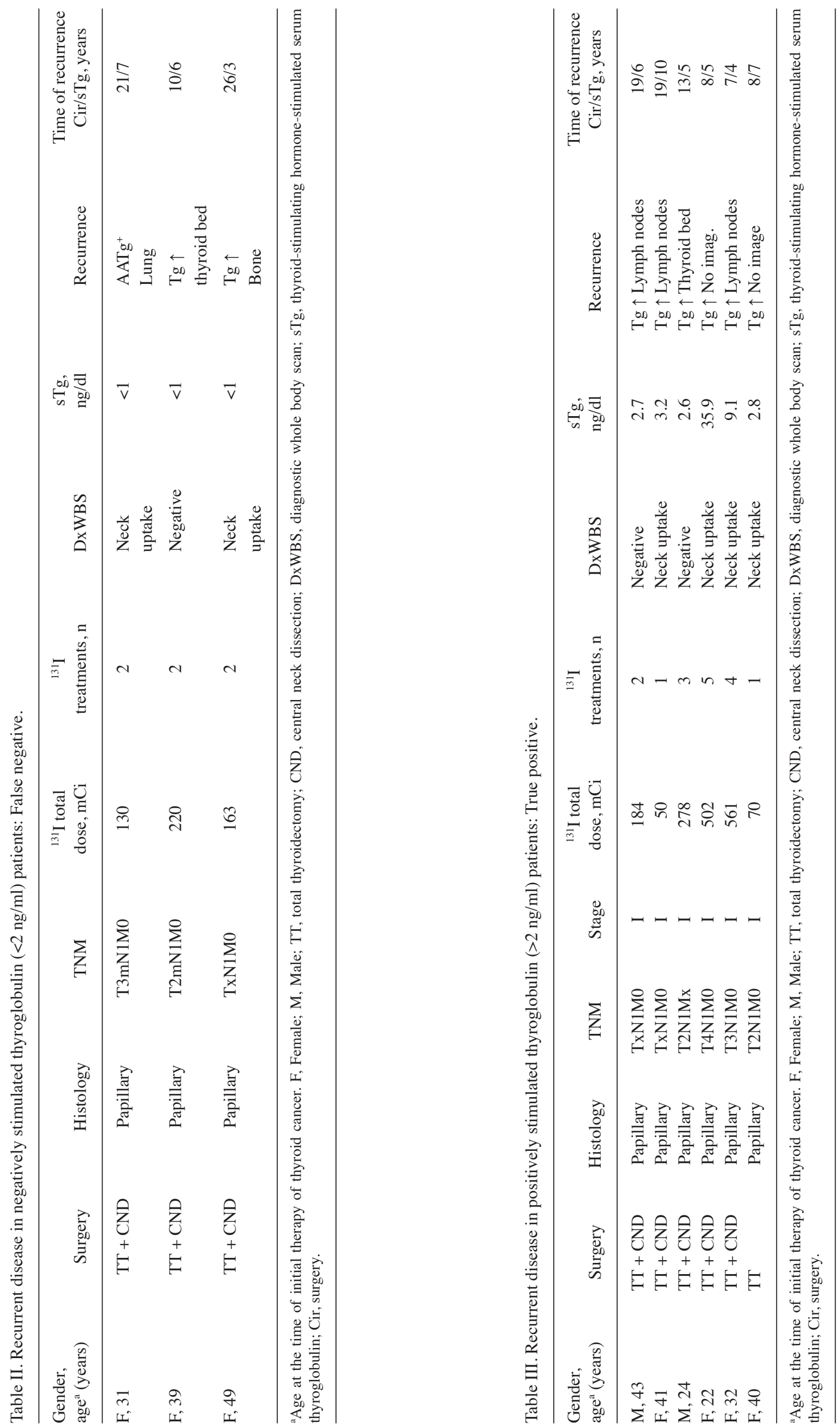
Table IV. Crosstable between NED and sTg level.

\begin{tabular}{|c|c|c|c|}
\hline & \multicolumn{2}{|c|}{ NED } & \multirow[b]{2}{*}{ Total } \\
\hline & Yes & No & \\
\hline \multicolumn{4}{|c|}{$\mathrm{sTg}, \mathrm{ng} / \mathrm{ml}$} \\
\hline$<2$ & $34(\mathrm{TN})$ & $3(\mathrm{FN})$ & 37 \\
\hline$>2$ & $6(\mathrm{FP})$ & 6 (TP) & 12 \\
\hline Total & 40 & 9 & $\mathrm{P}=0.001$ \\
\hline
\end{tabular}

Statistical analysis. Statistical analysis was performed using SPSS, version 20 (IBM, Armonk, NY, USA). Comparisons of the categorical data were performed using the $\chi^{2}$ test (Fisher's exact). $\mathrm{P}<0.05$ was considered to indicate a statistically significant difference. The results of the 49 patients were analysed for estimation of the accuracy of the rhTSH stimulating Tg levels. A 2x2 analysis was constructed of NED (yes or no) and this was compared to sTg levels (negative or positive with a cut-off value of $>2.0 \mathrm{ng} / \mathrm{ml}$ ). The stimulated $\mathrm{Tg}$ accuracy was assessed through sensitivity, specificity, positive predictive value (PPV) and NPV determinations.

\section{Results}

Population data. The clinical characteristics of the study population are reported in Table I. All patients received at least one ablation dose of ${ }^{131} \mathrm{I}$, nine patients (18.8\%) received two doses, and nine received three or more doses, with a mean dose of $160 \mathrm{mCi}$ (range, 50-670 mCi). The tumour stages are shown in Table I. Distant metastases were observed in one patient when the initial cancer diagnosis was determined; however, an excellent response to therapy was exhibited, resulting in negative ${ }^{131} \mathrm{I}$ WBS and serum supTg levels $<1.0 \mathrm{ng} / \mathrm{ml}$, and the patient was considered free of disease at the time of sTg evaluation. The time between initial surgery and the rhTSH stimulation test ranged from 1-36 years [mean \pm standard deviation $(\mathrm{SD}), 7.9 \pm 7.6$ years].

Follow-up and recurrence. The mean $( \pm \mathrm{SD})$ follow-up after the rhTSH stimulation test was 12.4 years $( \pm 0.7$ years), with a minimum of 10 years and a maximum of 13 years. In total, eight of the 49 patients were lost to follow-up, seven patients exhibited no evidence of disease and one exhibited recurrent disease at the time of the last appointment (5 years following the rhTSH stimulation test). Following the rhTSH stimulation test, $75.5 \%$ (37/49) of the patients with baseline Tg levels $<1.0 \mathrm{ng} / \mathrm{ml}$ exhibited no increase in sTg levels $(<2 \mathrm{ng} / \mathrm{ml})$. By contrast, 12 of the 49 patients $(24.5 \%)$ exhibited rhTSH-stimulated $\mathrm{Tg}$ levels $>2 \mathrm{ng} / \mathrm{ml}$. DxWBS following rhTSH administration was negative in 23 patients (46.9\%), revealed uptake in the thyroid bed in seven patients (14.3\%) and was not performed in 19 patients (38.8\%).

Stimulated Tg accuracy. In total, nine recurrences (18.4\% of patients) were identified during a mean of 12.4 years of

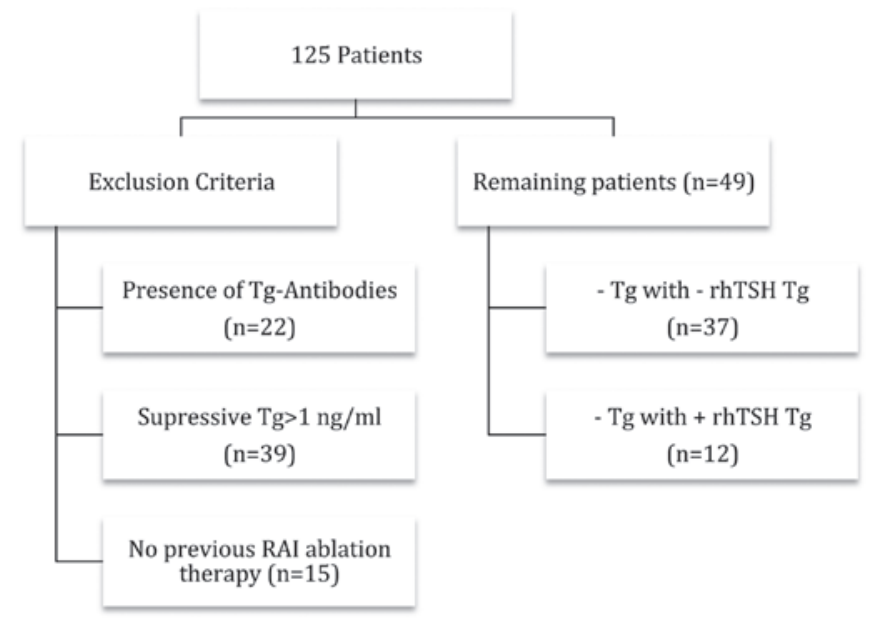

Figure 1. Flowchart for patients with application of exclusion criteria. Tg, thyroglobulin; rhTSH, recombinant human thyroid-stimulating hormone; RAI, radioactive iodine.

follow-up after the rhTSH stimulation test. Recurrence was detected after a median $( \pm \mathrm{SD})$ of 5.9 years $( \pm 2.0$ years $)$. These recurrences occurred in the thyroid bed in two cases $(22.2 \%)$, in the lateral neck lymph nodes in three cases $(30 \%)$, and in the lungs and bone in one case each (11.1\%). Additionally, two cases (22.2\%) with persistent elevated supTg levels were identified; however, no imaging or clinical evidence of disease was observed. All recurrences occurred in patients with papillary cancer and all of the patients exhibited lymph node metastasis (N1) at presentation. Of all the 49 patients, 22 had lymph node metastasis and $40.9 \%$ of these had a recurrence. The individual relapse time is shown in Tables II and III.

Among the 12 patients with sTg levels $>2 \mathrm{ng} / \mathrm{ml}$, only six patients experienced a recurrence [true positive (TP)], leading to a PPV of 50\%. Among the other 37 patients who exhibited negative sTg levels $(<2 \mathrm{ng} / \mathrm{ml}), 34$ patients did not experience any recurrence (true negative (TN)], leading to an NPV of $91.9 \%$. Three false negatives and six false positives (Table IV) were also identified. Analysing this data, a sensitivity of $66.6 \%$ and a specificity of $85.0 \%$ was estimated for the sTg levels.

\section{Discussion}

The upgrading of $\mathrm{Tg}$ assays has been associated with an improvement in diagnostic accuracy, leading to a lower prevalence of tumour recurrence than in the past, among patients declared free of disease (13). In the current retrospective study, the aim was to evaluate the accuracy of Tg-stimulated levels and its NPV and PPV in 49 patients subjected to total thyroidectomy and RAI ablation therapy with a long follow-up duration. A sensitivity of $66.6 \%$ and a specificity of $85 \%$ were obtained, with a PPV of $50 \%$ and an NPV of $91.9 \%$.

As described in a number of previous studies (13-15), rhTSH sTg may have a low sensitivity and low PPV. Results have been published indicating that $\sim 20 \%$ of patients who are clinically free of disease, with serum Tg levels $<1 \mathrm{ng} / \mathrm{ml}$ during suppression of $\mathrm{TSH}$, are likely to have a serum $\mathrm{Tg}$ 
Table V. Comparison between studies of accuracy of sTg levels following recombinant human TSH stimulation.

\begin{tabular}{|c|c|c|c|c|c|c|c|c|}
\hline $\begin{array}{l}\text { First author } \\
\text { (year) [ref.] }\end{array}$ & $\begin{array}{l}\text { Mean follow-up } \\
\text { time after sTg } \\
\text { test, years }\end{array}$ & $\begin{array}{l}\text { Time of } \\
\text { sTg test, } \\
\text { years }\end{array}$ & $\begin{array}{c}\text { sTg } \\
\text { cut-off } \\
(\mathrm{ng} / \mathrm{ml})\end{array}$ & $\begin{array}{l}\text { Recurrence } \\
\text { rate, } \%\end{array}$ & $\begin{array}{c}\text { Sensitivity, } \\
\%\end{array}$ & $\begin{array}{c}\text { Specificity, } \\
\%\end{array}$ & $\begin{array}{c}\text { Positive } \\
\text { predictive } \\
\text { value, } \%\end{array}$ & $\begin{array}{c}\text { Negative } \\
\text { predictive } \\
\text { value, } \%\end{array}$ \\
\hline Present study & 12.4 & 7.9 & $>2.0$ & 18.4 & 66.6 & 85.0 & 50.0 & 91.9 \\
\hline Brassard (2011) [4] & 6.2 & $0.75-1.0$ & $>1.4$ & 4.5 & 78.0 & 90.0 & 26.0 & 99 \\
\hline Kloos (2010) [13] & 7 & 5.5 & $>2.5$ & 17 & 80 & 97 & 84 & 95 \\
\hline Robbins (2002) [14] & 2 & $<2$ & $>2.0$ & 15.5 & 56.3 & 88.5 & 47.4 & 91.7 \\
\hline Schlumberger (2007) [18] & 2.33 & $0.75-1.0$ & $>0.9$ & $<3$ & $68-76$ & $81-91$ & NR & NR \\
\hline
\end{tabular}

sTg, TSH-stimulated serum thyroglobulin; TSH, thyroid-stimulating hormone; NR, not recorded.

levels $>2 \mathrm{ng} / \mathrm{ml}$ after rhTSH or thyroid hormone withdrawal at 12 months following surgery and RAI. In these patients, only one-third are likely to have an indication of persistent or recurrent disease and increasing Tg levels (7). Due to this, sTg may result in unnecessary additional testing and treatment in a number of patients. In the current study, only half of the patients with sTg levels $>2 \mathrm{ng} / \mathrm{ml}$ exhibited evidence of disease in the follow-up. Conversely, the NPV was high (91.9\%), allowing the identification of patients unlikely to experience disease recurrence and permitting less aggressive management strategies. These results are consistent with previously published results (Table V) (13).

In recent years, several studies have compared ultrasensitive Tg assays with sTg, suggesting similar NPVs (99 vs. 100\%) (16,17). In low-risk patients, it was suggested that TSH stimulation tests may be avoided when sensitive suppressive Tg levels are low $(<0.27 \mathrm{ng} / \mathrm{ml})$. In this study, $\mathrm{rhTSH}$ stimulation improved the positive predictive value of serum sTg determination only in patients with supTg levels $>0.27 \mathrm{ng} / \mathrm{ml}$, and the authors hypothesised that rhTSH must be restricted to such patients $(4,8)$. However, several studies using Tg assays with lower functional sensitivities have demonstrated that improved $\mathrm{Tg}$ sensitivity for disease detection is counterbalanced by an increase in false positives $(17,18)$.

When considering the DTC recurrence rate, long-term follow-up may demonstrate higher rates of tumour recurrence (13). This hypothesis was also described by Mazzaferi and Kloos (6), who reported locoregional recurrences and distant metastases (15 and $20 \%$, respectively) more than two decades following the initial therapy. The long follow-up period (12.4 years) is a significant factor in the current report, and allowed the confirmation that the recurrence rate remains high (18.4\%), even in a group of patients considered to be free of disease. Comparing this with the results published to date (Table V), a higher relapse rate was observed in the current study, which is likely to be due to the duration of follow-up, which was significantly longer than in the compared studies. In the current study, almost all the patients with recurrent disease were in TNM stage I (88.9\%) predominantly due to age $(<45$ years). The classification systems that use age to stratify risk may be inaccurate in predicting recurrence-free survival, as young patients have high recurrence rates (6). However, all patients with recurrences exhibited lymph node metastases (N1) at initial surgery, which is a well described risk factor for recurrent disease $(6,19)$. In the current study, the recurrence rate in patients with lymph node metastases was $40.9 \%$.

Pacini et al (20) suggested that neck US combined with sTg has the highest diagnostic accuracy for the detection of persistent disease, without the requirement for diagnostic WBS. In the current study, although $38 \%$ of the relapsed patients had not undergone DxWBS following rhTSH administration, a large number of false negatives in DxWBS were identified, leading to a low sensitivity and a low PPV of this diagnostic approach, confirming the results from other groups (21).

In the majority of published studies, Tg stimulation tests are performed within 1-2 years of surgery and ${ }^{131} \mathrm{I}$, considering that the majority of recurrences occur early following the primary treatment. By contrast, the current study includes patients whose primary treatment occurred within varied and occasionally long periods prior to Tg stimulation tests (median, 8.6 years; range, 1-36 years). Additionally, this study confirmed that late recurrences may occur in differentiated thyroid cancers, indicating that, even when varied and long periods of time following primary treatment have elapsed, the prognostic ability of the rhTSH stimulation test, particularly its NPV, is maintained.

In conclusion, the accurate surveillance for possible recurrence in patients considered to be free of disease is the predominant goal of long-term follow-up. With new sensitive serum Tg assays, rhTSH stimulation tests may not be routinely necessary. The benefit is greater in patients who have undetectable sTg levels (without serum Tg antibodies), allowing the identification of the patients who are free of disease. In these patients it is possible to ensure a more cost-effective and safe follow-up, monitoring supTg levels and performing neck ultrasound on an annual basis.

\section{References}

1. Jemal A, Siegel R, Ward E, et al: Cancer statistics, 2006. CA Cancer J Clin 56: 106-130, 2006.

2. Davies L and Welch HG: Increasing incidence of thyroid cancer in the United States, 1973-2002. JAMA 295: 2164-2167, 2006.

3. Hundahl SA, Fleming ID, Fremgen AM and Menck HR: A National Cancer Data Base report on 53,856 cases of thyroid carcinoma treated in the U.S., 1985-1995 [see commetns]. Cancer 83: 2638-2648, 1998

4. Brassard M, Borget I, Edet-Sanson A, et al: Long-term follow-up of patients with papillary and follicular thyroid cancer: a prospective study on 715 patients. J Clin Endocrinol Metab 96: 1352-1359, 2011.

5. Mazzaferri EL: An overview of the management of papillary and follicular thyroid carcinoma. Thyroid 9: 421-427, 1999. 
6. Mazzaferri EL and Kloos RT: Clinical review 128: Current approaches to primary therapy for papillary and follicular thyroid cancer. J Clin Endocrinol Metab 86: 1447-1463, 2001.

7. American Thyroid Association (ATA) Guidelines Taskforce on Thyroid N,Differentiated ThyroidCancer; CooperDS, Doherty GM, Haugen BR, et al: Revised American Thyroid Association management guidelines for patients with thyroid nodules and differentiated thyroid cancer. Thyroid 19: 1167-1214, 2009.

8. Schlumberger M, Borget I, Nascimento C, Brassard M and Leboulleux S: Treatment and follow-up of low-risk patients with thyroid cancer. Nat Rev Endocrinol 7: 625-628, 2011.

9. Castagna MG, Brilli L, Pilli T, et al: Limited value of repeat recombinant human thyrotropin (rhTSH)-stimulated thyroglobulin testing in differentiated thyroid carcinoma patients with previous negative rhTSH-stimulated thyroglobulin and undetectable basal serum thyroglobulin levels. J Clin Endocrinol Metab 93: 76-81, 2008.

10. Kloos RT and Mazzaferri EL: A single recombinant human thyrotropin-stimulated serum thyroglobulin measurement predicts differentiated thyroid carcinoma metastases three to five years later. J Clin Endocrinol Metab 90: 5047-5057, 2005.

11. Melo M, Costa G, Ribeiro C, et al: Stimulated thyroglobulin at recombinant human TSH-aided ablation predicts disease-free status one year later. J Clin Endocrinol Metab 98: 4364-4372, 2013.

12. Wittekind C, Compton CC, Greene FL and Sobin LH: TNM residual tumor classification revisited. Cancer 94: 2511-2516, 2002.

13. Kloos RT: Thyroid cancer recurrence in patients clinically free of disease with undetectable or very low serum thyroglobulin values. J Clin Endocrinol Metab 95: 5241-5248, 2010.

14. Robbins RJ, Chon JT, Fleisher M, Larson SM and Tuttle RM: Is the serum thyroglobulin response to recombinant human thyrotropin sufficient, by itself, to monitor for residual thyroid carcinoma? J Clin Endocrinol Metab 87: 3242-3247, 2002.
15. Mazzaferri EL and Kloos RT: Is diagnostic iodine-131 scanning with recombinant human TSH useful in the follow-up of differentiated thyroid cancer after thyroid ablation? J Clin Endocrinol Metab 87: 1490-1498, 2002.

16. Giovanella L, Ceriani L, Ghelfo A, et al: Thyroglobulin assay during thyroxine treatment in low-risk differentiated thyroid cancer management: comparison with recombinant human thyrotropin-stimulated assay and imaging procedures. Clin Chem Lab Med 44: 648-652, 2006.

17. Smallridge RC, Meek SE, Morgan MA, et al: Monitoring thyroglobulin in a sensitive immunoassay has comparable sensitivity to recombinant human tsh-stimulated thyroglobulin in follow-up of thyroid cancer patients. J Clin Endocrinol Metab 92: 82-87, 2007

18. Schlumberger M, Hitzel A, Toubert ME, et al: Comparison of seven serum thyroglobulin assays in the follow-up of papillary and follicular thyroid cancer patients. J Clin Endocrinol Metab 92: 2487-2495, 2007.

19. Nascimento C, Borget I, Al Ghuzlan A, et al: Persistent disease and recurrence in differentiated thyroid cancer patients with undetectable postoperative stimulated thyroglobulin level. Endocr Relat Cancer 18: R29-R40, 2011.

20. Pacini F, Molinaro E, Castagna MG, et al: Recombinant human thyrotropin-stimulated serum thyroglobulin combined with neck ultrasonography has the highest sensitivity in monitoring differentiated thyroid carcinoma. J Clin Endocrinol Metab 88: 3668-3673, 2003.

21. Torlontano M, Crocetti U, Augello G, et al: Comparative evaluation of recombinant human thyrotropin-stimulated thyroglobulin levels, 131I whole-body scintigraphy, and neck ultrasonography in the follow-up of patients with papillary thyroid microcarcinoma who have not undergone radioiodine therapy. J Clin Endocrinol Metab 91: 60-63, 2006. 